\title{
The Study of Cross Blowing to Eliminate the Slag of Bulk Amorphous Alloy During Laser Cutting
}

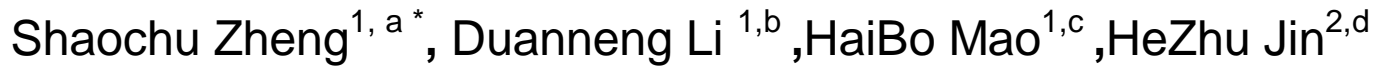 \\ ${ }^{1}$ Guangdong University of Technology, Mechanical Engineering Institute, GuangZhou,GuangDong \\ 510006,China ; \\ ${ }^{2}$ PrometalTech Co.Ltd, Dongguan, Guandong , 523600, China

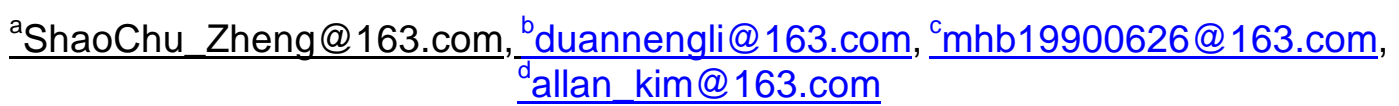

Keywords: amorphous alloy laser cutting cross blowing slag

Abstract. Bulk Amorphous alloy generates slag at the bottom of the cutting section during laser cutting, which decreases the quality of the cutting process. It's very difficult to be cleared with adjusting the parameters of the cutting machine only. The paper takes the pattern of cross nitrogen blowing during laser cutting on the workpiece's bottom face, through changing its angles and pressures of blowing to study the effect of different angles and pressures on eliminating the generated slag. And take the compressed air as comparison to study the difference of reactant gas and non-reactant gas on eliminating the slag. Using ANSYS FEM software to analysis the gas distribution, and it comes almost the same conclusion that the cross blowing can efficently deduce the slag. The study gives an pratical and theoretical foundation to eliminate the slag and improve the cutting quality.

\section{Introduction}

Amorphous Alloy is a new alloy whose atoms were arranged topological disorder in three-dimensional solid state and remain relatively stable in a certain temperature range.Comparing to the traditional crystalline alloy, amorphous alloys have completely different properties, including low melting point, high strength, high toughness and high wear resistance, and so on,making it gradually applied in people's daily consumer goods, such as using high wear resistance of the preparation of amorphous alloy audio video head, golf clubs, luxury watches, fishing pole, etc[1].

The amorphous products were produced by die casting with a multi-mode cavity,and then be separated from the framework with laser cutting.It demands the cutting surface should be smooth, the bottom of the cutting section has not slag and the cutting section keeps non-crystalline structure.This puts forward quite a high requirement and difficulty to laser cutting.During the cutting process, the alloy was heated to melt, and the melten material flows downward along the kerf at the injection of the assit gas, due to the cooling effect of the gas, as well as the sticky and the surface tack of the material, slag generates at the end of the cutting section, as was shown in Fig.1.

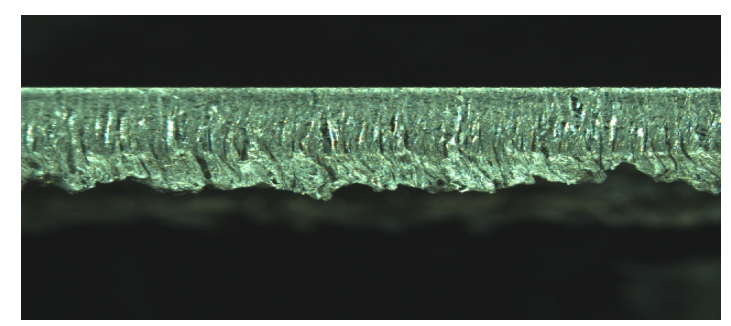

Fig. 1 the slag ont the cutting section

Fig. 1 shows that the maximum length of the slag is at almost half of the product' $s$ thickness. This seriously affects the laser cutting quality and also increase the difficulty and workload of its post-processing. After a lot of experiments, we found that by adjusting the cutting process parameters can only get brittle and loose, easy go slag, but can't achieve the purpose of elimination. This paper aims to explore a process method to eliminate the slag in order to improve the quality of the surface 
during laser cutting. In the experiment, we took Zr-based amorphous alloy as the research object, using fiber laser cutting equipment, supplemented by the cross blowing towards to the bottom of the workpiece at the angle of $45^{\circ}$ and $90^{\circ}$ to explore the cross blowing process for the effect of eliminating the generated slag. Two kinds of gas -nitrogen and compressed air -were taken in the experiment to explore the impact of non-oxidizing and oxidizing gas in eliminating slag. And then use ANSYS FEM software to further simulation the distribution of the airflow situation on the bottom of the workpiece and analyze its mechanism.

\section{Slag formation mechanism and its influencing factors}

The formation mechanism of laser cutting bulk amorphous alloy.Technology research of laser cutting on bulk amorphous alloy, at present, can not find the relevant reports at home and abroad. Amorphous alloy belongs to non-ferrous metal, with low melting point and higher characteristic of laser reflectivity, laser absorption rate is very low, and viscosity is bigger after molten material cooling down which determines the difficulty of the laser processing.

The formation of amorphous alloy needs a suitable glass forming temperature and liquidity. According to different cutting materials and auxiliary gases, laser cutting has divided into four types :vaporization cutting, molten cutting, oxygen cutting and fracture control cutting. Through a large number of amorphous alloy cutting experiments, XRD ray scanning was carried out on the cutting section, as shown in Fig.2. Scanning results showed that the section material remained amorphous organization. This shows that temperature of laser cutting is within glass forming temperature, and the cutting of amorphous alloy belongs to molten cutting.

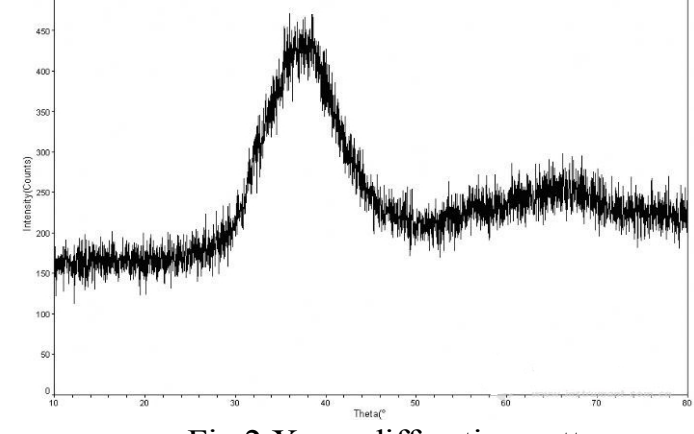

Fig.2 X-ray diffraction pattern

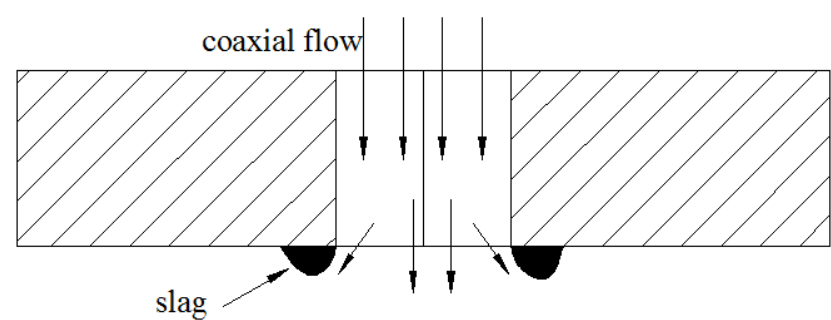

Fig.3 slag formation schematic

Due to the cooling effect of the coaxial jetting gas, the molten material is cooling rapidly in the process of outflow slitting, then some adhere on the cutting section and forming the rough surface, some are exclude by the gas. As the high pressure gas belongs high-speed stream, when reaching the bottom corner of the workpiece, airflow outwards[2], pushing partial melting material adhere to the sides of the kerf to form a slag which was shown in Fig.3.

Factors affecting the slag.Bulk amorphous laser cutting showed that the amount of the slag on the bottom was greatly influenced by the cutting frontier temperature and the pressure of the coaixal gas. When the cutting frontier temperature is higher,the viscosity of the molten material will be decreased and the molten material can be easily excluded by the gas; when the coaxial gas pressure increases, the capacity of it to exclude the molten material enhanced.It was also found that the frontier temperature of the laser cutting related to the energy acting on the material, and when the energy establish thermal equilibrium, the frontier temperature remained relatively stable;also, the pressure was limited by the supply equipment, and when the pressure is too large ,there will generate shock wave between the nozzle and the workpiece surface, which will reduced the capacity of the gas to exclude the slag[3].From all the experiments we found that an non-slag section can't be obatained only by adjusting the processing prameters. So the paper carried out the flowing expriment 


\section{Cross blowing Experiment}

Cutting materials and process equipment. The experiment material took bulk Zr-based amorphous alloy $\mathrm{Zr}_{57} \mathrm{Nb}_{5} \mathrm{Al}_{10} \mathrm{Cu}_{15.4} \mathrm{Ni}_{12.6}$ with the size of $75 \times 10 \times 1 \mathrm{~mm}$. Before the experiment, the material for deburring, degreasing,surface roughness and other treatment to clear the structure defects,its physical properties was shown in Tab.1.

\begin{tabular}{lll}
\hline Item & value & unit \\
\hline density & 6.66 & $\mathrm{~g} / \mathrm{cm} 3$ \\
melting point & 1092 & $\mathrm{~K}$ \\
\hline \multicolumn{2}{l}{ Tab. 1 the physical properties of Zr57Nb5A110Cu15.4Ni12.6 }
\end{tabular}

From cost considerations, the experimental non-oxidizing gas selected relatively low-cost nitrogen, gas purity is $99.99 \%$,selected compressed air as oxidation reaction gas ,and make comparation to explore its impact on the elimination of the slag. The corresponding physical properties of the two gases are shown in Tab.2.

\begin{tabular}{|c|c|c|}
\hline Item & nitrogen & Compressed air \\
\hline density ( kg/m3 ) & 1.123 & 1.161 \\
\hline dynamic viscosity $\mathrm{ukg} /(\mathrm{s} . \mathrm{m})$ & 17.89 & 18.54 \\
\hline
\end{tabular}

Tab. 2 the physical properties of $\mathrm{N} 2$ and compressed air

The cutting equipment chose IPG's YLR-150/1500-QCW-MM-AC Single-mode Yb-doped fiber laser device, which laser wavelength is $1070 \mathrm{~nm}$ and the maximun output power is $1500 \mathrm{~W}$.Its focal length is $100 \mathrm{~mm}$, the light spot diameter of the focal position is about $50 \mu \mathrm{m}$, the beam mode is $\mathrm{TEM}_{00}$ and the beam quality $\mathrm{M}^{2}<1.05$.

Experimental method and measurement equipment. As was elaborated above,the experiment takes the method of cross blowing to blow the melting meltal to the rear side to get a non-slag section. During cutting, the workpiece was fixed on the positioning fixture, blowing nozzle was placed on the buttom of the workpiece, which has two position at the angle of $\theta=45^{\circ}$ and $\theta=90^{\circ}$.changes the gas pressure $\mathrm{q}_{2}, \mathrm{q}_{2}=0.3 \sim 1.6 \mathrm{MPa}$. The whole apparatus placed on CNC platform , using the outside light into the single-channel approach to cutting. The experimental method is schematically shown in Fig.4.

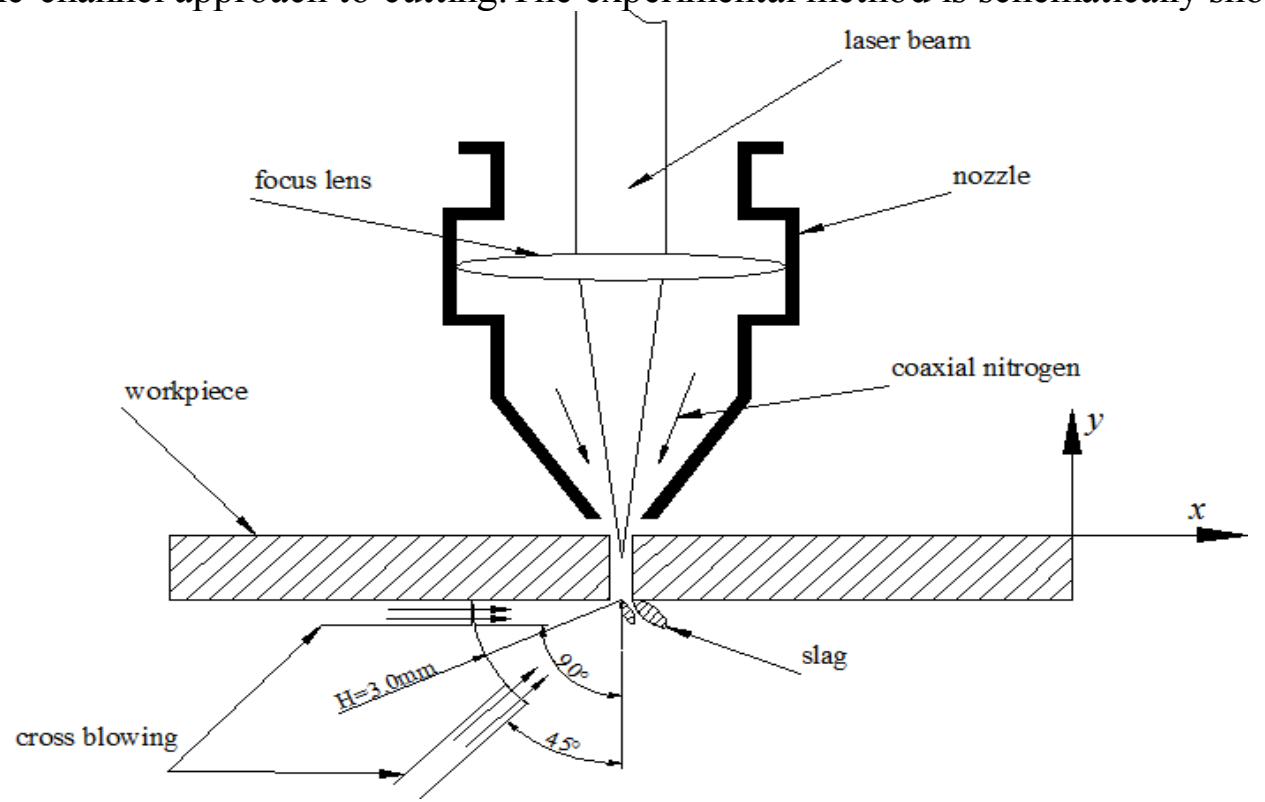

Fig.4 the experimental method schematic 
The section side of the blowing nozzle is a flat blowing channel which size is $12 \times 0.8 \mathrm{~mm}$, when cutting, the blowing gas can cover the whole cutting section, the distance of the nozzle and the cutting surface is $\mathrm{H}=3.0 \mathrm{~mm}$.

Measuring equipment selects Seven Ocean Electric co., LTD.’s SOV - 1010 M imaging surveying and mapping instrument to measure the result of the experiment, the display resolution of the machine is $0.0001 \mathrm{~mm}$.

\section{Experimental Porcedure and Results}

Taking laser power $\mathrm{p}=450 \mathrm{~W}$, standoff distance ( distance between the nozzle and the workpiece ) $\Delta \mathrm{h}=0.4 \mathrm{~mm}$, cutting speed $\mathrm{v}=20 \mathrm{~mm} / \mathrm{s}$, coaxial assit nitrogen gas pressure $\mathrm{q}_{1}=1.3 \mathrm{MPa}$ and the cross blowing is synchronized with the laser axis. Experiments using single factor method, changing one parameter and keep the others to guide the optimization of experimental parameters.

The angle of blowing took $\theta=45^{\circ}$ and $\theta=90^{\circ}$ and divided into two groups with the compressed air and nitrogen. We adjusted the blowing pressure $\mathrm{q}_{2}$, observed and recorded the length of the slag after blowing and then analyzed it. The measure of the slag started from its root and took ten bovious heights as the measurements and then took its averages as its final length. The results was shown in the Fig. 5.

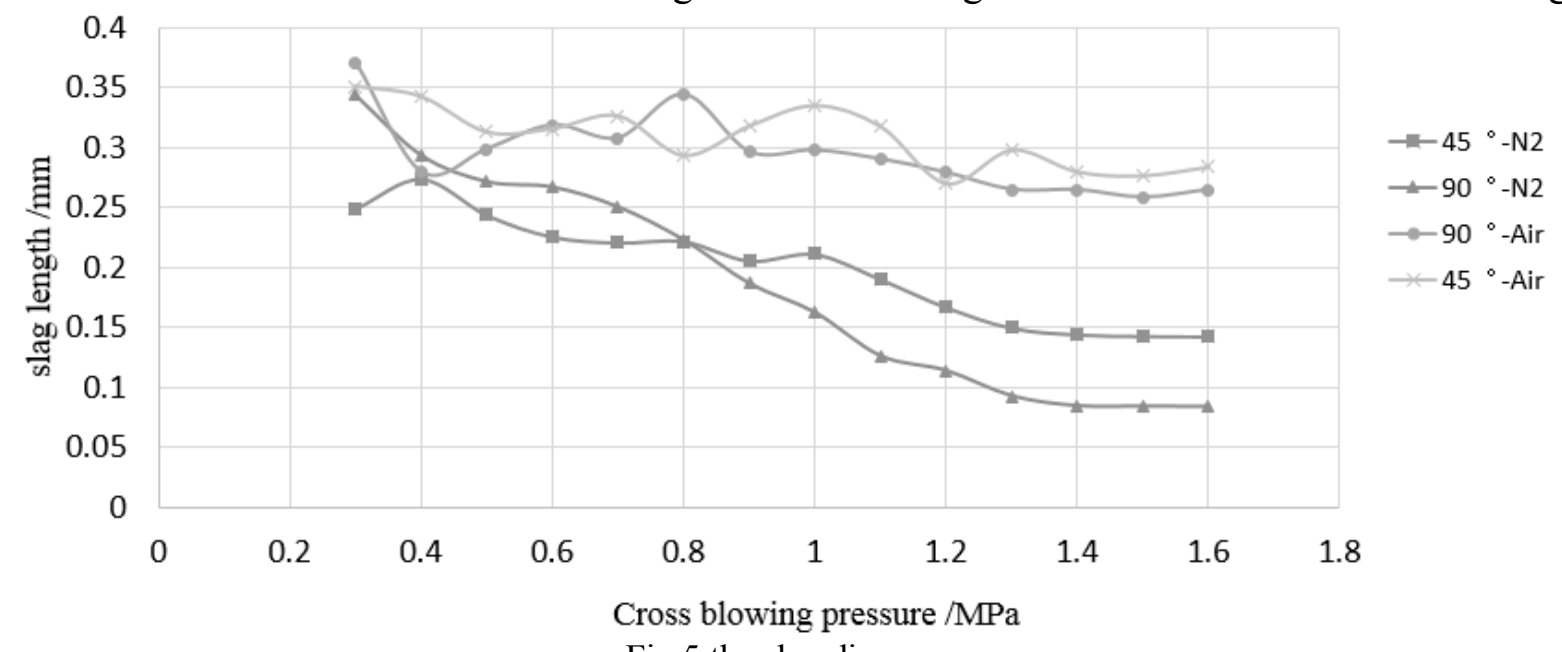

Fig. 5 the slag diagram

The experiments shown that the cross blowing could mostly blow the slag into the designated direction and eliminate the slag which was facing the blowing.From Fig. 5 we can found, when blowing the compressed air,the length of the slag is quite fluctuant and can't be completely eliminated.That's because the oxygen in air can easily make a chemical reaction with the molten material which is blowing out from the kerf, such as

$$
\begin{array}{r}
\mathrm{Zr}+\mathrm{O}_{2}=\mathrm{ZrO}_{2} \\
2 \mathrm{Cu}+\mathrm{O}_{2}=2 \mathrm{CuO}
\end{array}
$$

ect[4],making the slag and the adhesion strength increased at the bottom of the workpiece.As the gas pressure, chemical reaction go stable gradually, the slag eventually keep a certain length.As was shown in Fig.5,the slag lengths at the angle of $\theta=45^{\circ}$ and $\theta=90^{\circ}$ were stable at about $\mathrm{L}=0.276 \mathrm{~mm}$ and $\mathrm{L}=0.253 \mathrm{~mm}$. But nitrogen gas is almost not involved in reaction[5], the slag length is relatively stable .The slag lengths under the two blowing angles respectively keep in about $\mathrm{L}=0.124 \mathrm{~mm}$ and $\mathrm{L}=0.083 \mathrm{~mm}$, the nitrogen is more effective than the compressed air to eliminate the slag.

Adjusting the blowing angle,we can found from the constract of the results that blowing at $\theta=90^{\circ}$ is better than at $\theta=45^{\circ}$. Assuming that the gas blows to the bottom at the angle $\theta\left(0^{\circ}<\theta \leq 90^{\circ}\right)$, there exist a shear stress $\tau_{x}$ at the direction of $x$ axis in the vertical plane of the laser beam and the 
workpiece, which blow the molten metal to the rear side.Assuming the actual area the gas acts on the molten metal is $S_{\text {eq }}$, air damping is $c$, keep the blowing angle $\theta$ and distance $H$, then the equivalent pressure $\mathrm{q}$ acting on the molten metal is :

$$
q=c \cdot q_{2}
$$

the force acting on the molten matal is:

$$
\mathrm{F}=\mathrm{q} \cdot \mathrm{S}_{\mathrm{eq}}
$$

And the shear stress is :

$$
\tau_{x=} \mathrm{F} \cdot \sin \theta
$$

As the formulation(3) shows, the shear stress $\tau_{x}$ changes with the blowing angle $\theta$ in sine law within $\theta\left(0^{\circ}<\theta \leq 90^{\circ}\right), \tau_{x}$ increases when $\theta$ increases and it get the theoretical maximum at the angle of $90^{\circ}$,that will be easy to eliminate the slag.

By Fig.5, the minimum slag length can be obtained in blowing nitrogen at the angle of $90^{\circ}$ with the pressure of $1.4 \mathrm{MPa}$, the minimum length is $0.083 \mathrm{~mm}$, and the section is shown in figure 6 , which has almost non-slag on the section.

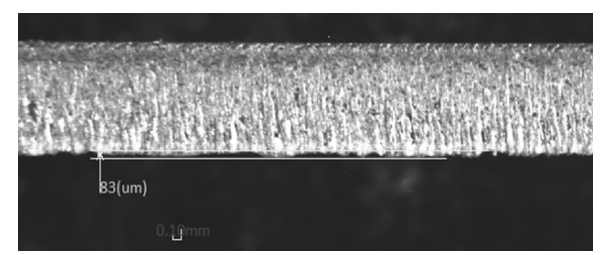

Fig.6、 $\theta=90^{\circ}$ the best effect of blowing N2

\section{FEM The numerical simulation analysis}

In the process of laser cutting, the alloy under high temperature laser irradiation melts to form a molten stream which has a certain viscosity. It's generally considered[3] that in the kerf the fluid is in laminar or an uncertain state that laminar turns to turbulent flow. This uncertainty has led to cut flat uneven, and the presence of large amounts of slag. Cross blowing assist gas, can increase the stability of the flow field at the bottom and obtain relatively high cutting quality of the cutting surface.

During the laser cutting, the nozzle diameter is bigger than the diameter of incision, airflow area is an intersection of the rectangular of the incision and the semi-circular of the nozzle. For this kind of cross section shape, equivalent diameter can be calculated based on the ratio of the quadruple of the cross-sectional area and the cross-sectional perimeter[6]. The simulation takes RNG k- $\square$ turbulence model, using the finite element software ANSYS to simulate the flow field at the bottom. The grids take unstructured grids, the gas model is simplified to two-dimensional axisymmetric compressible flow which contains only nitrogen and oxygen. The computational domain select the pressure inlet on the nozzle, giving the total pressure, total temperature and velocity, set the outlet, invironment pressure, temperature, use symmetrical axis condition and the boundary is set into the no reflection boundary[7].The simulation results are shown in Fig.7 and Fig.8.

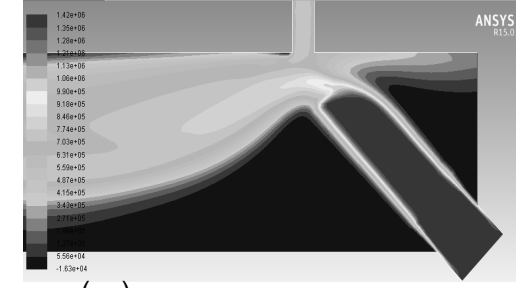

Fig. $7^{(a)}(\mathrm{a})$ the pressure icloud image of the angle about $45^{\circ}$

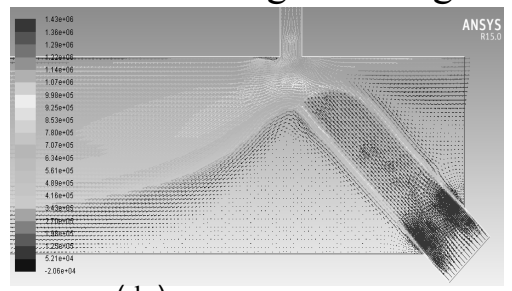

(b) vectors distribution

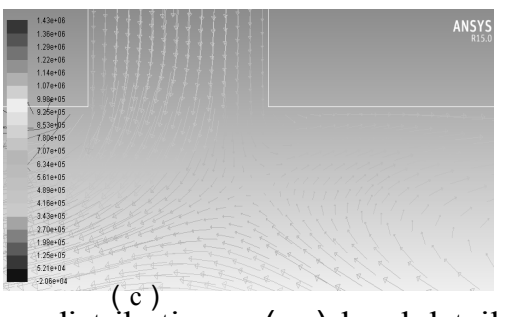

, ( c ) local details 

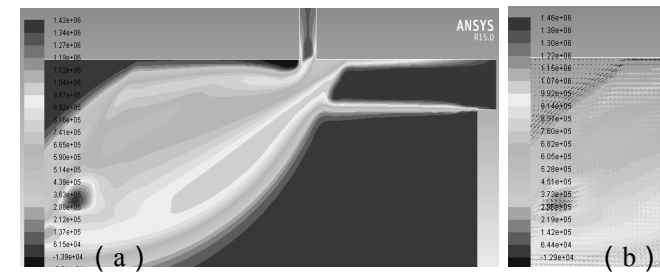

Fig. 8 ( a ) he pressure icloud image of the angle about $90^{\circ}$
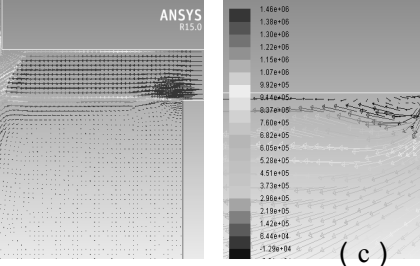

(b) vectors distribution, ( c ) local details

Fig. 7 shows the gas flow field distribution at $\theta=45^{\circ}$ and $\mathrm{q}_{2}=1.4 \mathrm{MPa}$. From the stress cloud picture Fig. 7 (a), the pressure of the coaxial flow along the kerf was significantly decreased, that' $\mathrm{s}$ because part of the cross flow swam upward and offset a part pressure of the coaxial gas, thus cause the flow pressure reduced, From Fig.7 (c) you can see, the slag location, part of the gas spin to outside corner and the slag effect is not ideal.

Fig. 8 shows the gas flow field distribution at $\theta=90^{\circ}$ and $\mathrm{q}_{2}=1.4 \mathrm{MPa}$. From Fig. 8 (c), we can found that the degree of the laminar in the horizontal direction is more higher, the two streams collide at the junction and the cross flow generates transverse shear flow and slag effect is obvious. While on the left corner, there is an obvious swirl gas flow, cause the residue accumulates on the left side, namely slag accumulates on the left and the front (facing the flow) almost has no slag accumulation.

Therefore, from the above gas flow field simulation diagrams we can see, cross gas flow collides with the coaxial gas which generates not only the flow to eliminate the slag but also a part of the swirl flow and a part of upstream flow along the kerf. This will cause instability to the coaxial flow and reduce the effect of slag. When blowing in the angle of $90^{\circ}$, crosscutting gasflow acts significantly, and will not affect the coaxial flow, slag effect is very obvious.

\section{Conclusions}

Bulk amorphous alloy has such advantages as low melting point, high hardness and brittleness, Laser cutting is non-contact, flexible and won't produce damage to alloy, what's more, it won't destroy the non-crystalline structure of the cutting section, having incomparable advantage over traditional machining.

Taking cross blowing nitrogen at the bottom of the workpiece can effectively eliminate the generation of slag during laser cutting, and improve the quality of laser cutting on amorphous alloy. At the same time, compared to blow compressed air, it is concluded that nitrogen can be more effective to eliminate the slag. For other non-ferrous bulk metal, the mechanism of generating slag is similar to that of amorphous alloy, and the method explored in this paper is also applicable.

Through the experiment and FEM simulation, we get the conclusion that cross blowing at the angle of $90^{\circ}$, the blowing pressure $\mathrm{q} 2=1.4 \mathrm{MPa}$ can get almost no slag cutting section.

\section{Reference}

[1] ChuanFu Li, ChuanJiang Zhang, XueXiang Xin. Journal of shandong institute of light industry, 2008,3.22 (1) : 50-53(In Chinese)

[2] Lei Hong, ChengLong Mi, LiJun Li. Journal of optics, 2008, 3 (28) : 522-527. (In Chinese)

[3] Cuo Yan,LiJun Li, Juan Li. Laser Technology, 2005.29 (3): 270-274

[4]XinXin Xie, KaiWu, DaiGang Nie, etc. Journal of electrochemistry, 2003.5, 9 (2) : 147-155 (In Chinese)

[5] Lianjun Chen, DeGuo Ma. Journal of mechanical engineering and automation, 2004.10, then 9-82(In Chinese)

[6] HuiLin Chen, GuangPo Zhao, RuShan Bie,etc. Journal of engineering thermal physics, 1999.20 (5) : 627-631(In Chinese) 
[7] ZiNiu Wu. The basic principle of computational fluid mechanics [M]. Beijing: science press, 2001.42-44(In Chinese) 